\title{
COOLING CURVE ANALYSIS IN BINARY AL-CU ALLOYS: PART II- EFFECT OF COOLING RATE AND GRAIN REFINEMENT ON THE THERMAL AND THERMODYNAMIC CHARACTERISTICS
}

\author{
Mehdi Dehnavi , Mohsen Haddad-Sabzevar, Mohamad Hasan Avazkonnadeh- \\ Gharavol
}

Department of Materials Engineering and Metallurgy, Ferdowsi University of Mashhad, Mashhad, Iran.

Received 25.04.2015

Accepted 25.08.2015

\begin{abstract}
The Al-Cu alloys have been widely used in aerospace, automobile, and airplane applications. Generally Al-Ti and Al-Ti-B master alloys are added to the aluminium alloys for grain refinement. The cooling curve analysis (CCA) has been used extensively in metal casting industry to predict microstructure constituents, grain refinement and to calculate the latent heat of solidification. The aim of this study is to investigate the effect of cooling rate and grain refinement on the thermal and thermodynamic characteristics of Al-Cu alloys by cooling curve analysis. To do this, $\mathrm{Al}-\mathrm{Cu}$ alloys containing 3.7, and $4.8 \mathrm{wt} . \% \mathrm{Cu}$ were melted and solidified with 0.04 , $0.19,0.42$, and $1.08 \mathrm{~K} / \mathrm{s}$ cooling rates. The temperature of the samples was recorded using a $\mathrm{K}$ thermocouple and a data acquisition system connected to a PC. Some samples were Grain refined by Al-5Ti-1B to see the effect of grain refinement on the aforementioned properties. The results show that, in a well refined alloy, nucleation will occur in a shorter time, and a undercooling approximately decreases to zero. The other results show that, with considering the cooling rate being around $0.1{ }^{\circ} \mathrm{C} / \mathrm{s}$, the Newtonian method is efficient in calculating the latent heat of solidification.

Key words: Thermal Analysis, Latent Heat, Computer-Aided Cooling Curve Analysis, Grain Refinement
\end{abstract}

\section{Introduction}

Depending on the casting conditions and alloy composition, microstructure and properties of the different aluminium alloys will be different [1]. It is well known that metals and alloys usually solidify with coarse columnar grain structure under normal

*Corresponding author: M. Dehnavi,Mehdi_6664@yahoo.com 
casting conditions unless the mode of solidification is carefully controlled. It is possible to develop fine equiaxed grains in the as cast structure either by increasing the number of nucleation sites or by grain multiplication $[1,2]$. The most widely accepted method is to add commercial Al-5Ti-1B master alloys into the melt in the form of rod [2]. The thermal analysis is widely used in the evaluation of processing of aluminum alloys because it can provide several pieces of information regarding the alloy. Thermal analysis is based on the fact that the thermal events on a heating or cooling curve are directly related to phase transformation occurring in a sample. Depending on the nature of the analysis, different kinds of information such as the latent heat of solidification, solid fraction, and amounts and types of phases that solidify can be determined. Many techniques are available to investigate the solidification of metals and alloys. Some of them were standardized such as DTA and DSC. Although these techniques are very accurate and well documented, they are inadequate for industries to investigating solidification of metals and alloys. Because it's required to use very small samples and they need expensive facilities and technical expertise [3,4]. The other way for investigating solidification of metals and alloys is the cooling curve analysis method. This technique is based on recording and analysis of the temperature versus time data collected during the solidification of the sample. In recent years computer-aided cooling curve analysis (CA-CCA) has been used to determine thermo-physical properties of alloys, latent heat and solid fraction. Because it's very simple to setup, it can be widely used, especially in industries [5-7]. It just needs to place a thermocouple in the melt and allow the melt to solidify while the temperature is recorded as a function of time. The cooling curve does not always indicate all the reactions occurring during solidification of a casting clearly, due to the small amounts of heat evolved by certain phase transformations, so more sensitive techniques should be developed. It has been found that the first derivative of the cooling curve can be employed to emphasize small heat effects not resolved on the cooling curve itself [7,8]. The use of first derivatives improves the accuracy of determination of the characteristic features of the cooling curve. A critical requirement for the CCA is determination of what is called "the zero curve" or "the base line". The zero curve, in principle, is the derivative of a cooling curve, if there is no phase transformation [7,9]. To calculate the latent heat by CCA, two methods are employed: the Fourier and the Newtonian methods. The advantage of Newton's method is its simplicity. Because we have used it in the current research, the basic principles are discussed here briefly. This model assumes Newtonian cooling of the sample, i.e. no thermal gradients across the sample, and heat transfers by convection to the mold. As the enthalpy decrease in metal is equal to the heat transferred to the mold the mathematical form of the Newtonian analysis is given by the following equations [8-12].

$$
\frac{d Q_{L}}{d t}-V \rho C_{p} \frac{d T}{d t}=h A\left(T-T_{0}\right)
$$

Where: $V, A, T_{0}, Q_{L}, \rho, h, C_{P}, T$, and $t$ are volume of the sample, surface area, ambient temperature, latent heat of solidification, density of metal, heat transfer coefficient, specific heat of the metal, temperature, and time, respectively. The first term on the left hand side of the Eq. (1) is the heat generated by phase transformation and the second term is heat lost by the metal, and the term on the right hand side of the Eq. (1) 
is heat transferred to the mould. Rearranging Eq. (1), and considering the absence of phase transformation $\left({ }^{d Q / d t=0}\right)$ Eq. (2) is obtained for the zero curve:

$$
\frac{d T}{d t}=-\frac{h A\left(T-T_{0}\right)}{V \rho C_{p}}
$$

Using the notations: " $c c$ " for cooling curve and " $z c$ " for zero curve, one can calculate the rate of heat released during the phase transformation as:

$$
\frac{d Q_{L}}{d t}=V \rho C_{p}\left[\left(\frac{d T}{d t}\right)_{C C}-\left(\frac{d T}{d t}\right)_{Z C}\right]
$$

Integrating Eq. (3) will give:

$$
Q_{L}=V \rho C_{p} \int_{0}^{t_{s}}\left[\left(\frac{d T}{d t}\right)_{C C}-\left(\frac{d T}{d t}\right)_{Z C}\right] d t
$$

Where $t_{S}$ is the solidification time and L is "Latent Heat" as defined below:

$$
L=\frac{Q_{L}}{V \rho}
$$

$\mathrm{L}=\mathrm{C}_{\mathrm{p}}$ (area under the curve derived from cc - area under the curve derived $\mathrm{zc}$ )

Where $\mathrm{L}$ is the latent heat.

\section{Experimental procedure}

Two binary Al-Cu alloys were used in this study which their chemical composition is given in Table 1. Two sets of solidification tests were performed as follows. In the first set, the effect of grain refinement on the solidification behaviour of $\mathrm{Al}-\mathrm{Cu}$ alloys was studied. To do this, $200 \mathrm{~g}$ of each alloy were melted in a graphite crucible by an electric furnace. After some preliminary experiments superheat temperature was selected as $740^{\circ} \mathrm{C}$ to reduce its harmful effects. Commercially available Al-5Ti-1B in the form of rod was used as grain refined which was added to the melt at the last stage of melting (about $0.1 \%$ of Al-5Ti-1B). For homogenous distribution of the grain refiner in the melt, it was stirred with and alumina rod and held in $720^{\circ} \mathrm{C}$ for $5 \mathrm{~min}$. The melt was then cast in a $100-200^{\circ} \mathrm{C}$ preheated sand mold $[13,14]$. To check the effectiveness of the grain refinement, the undercooling criteria as shown in Fig. 1 was used. The second set of experiments was designed to investigate the effect of cooling rate on the solidification processing of $\mathrm{Al}-\mathrm{Cu}$ alloys. Specimens with dimensions $(\mathrm{D} \times \mathrm{H}) 22 \times 20 \mathrm{~mm}$ and $\sim 10 \mathrm{~g}$ weight were melted and solidified in a DTA furnace. High purity argon gas (99.999\%) was running throughout the test to reduce the risk of oxidation of the samples. The samples were solidified with controlled conditions as indicated in Table 2. The temperature of the samples was recorded by a $\mathrm{K}$ thermocouple with the wire diameter of $0.15 \mathrm{~mm}$ placed at the center of the sample and 
an Advantech 4718 data acquisition system connected to a personal computer. Each trial was repeated twice. It should be noted that the thermocouple was calibrated with pure aluminum $(99.99 \%)$ before the tests. There are different ways to define the cooling rate. In the present research the cooling rate was calculated by dividing the total solidification range $\left(T_{L}-T_{S}\right)$ to the local solidification time according to [15]. The latent heat of solidification were calculated by numerical integration the area between the first derivative and zero curves based on the method reported by Yeh et. al. [16]. The fraction of the solid were calculated by using the Scheil equation as below:

$$
f=1-\left\lceil\left.\frac{T_{m}-T}{T_{m}-T_{p}}\right|^{\frac{-1}{2-K_{9}}}\right.
$$

Where $f, T_{m}, T, T_{0}$, and $k_{0}$ are solid fraction, melting temperature of pure Al, temperature, liquidus temperature, and partition coefficient respectively. Samples for study the microstructure were taken from a location close to the thermocouple tip. After grinding, polishing and etching with Keller's $\left(10 \mathrm{ml} \mathrm{HF}, 20 \mathrm{ml} \mathrm{HNO}_{3}, 20 \mathrm{ml} \mathrm{HCl}\right.$, and $50 \mathrm{ml}$ distilled water) and Weck's reagents, the microstructure were examined by an Olympus BX60 optical microscope.

Table 1. Chemical composition of the used alloys

\begin{tabular}{llll}
\hline $\mathrm{Cu}$ & $\mathrm{Si}$ & $\mathrm{Fe}$ & $\mathrm{Al}$ \\
\hline 3.7 & 0.04 & 0.06 & Bal. \\
4.8 & 0.05 & 0.06 & Bal.
\end{tabular}

Table 2. Samples coding

\begin{tabular}{|c|c|c|c|c|}
\hline $\begin{array}{l}\text { Sample } \\
\text { Code }\end{array}$ & $\begin{array}{l}\text { Test } \\
\text { Set } \\
\end{array}$ & $\begin{array}{l}\text { Cu Content } \\
\text { (wt.\%) }\end{array}$ & $\begin{array}{l}\text { Cooling } \\
\text { rate }(\mathrm{K} / \mathrm{s})\end{array}$ & $\begin{array}{l}\text { Grain } \\
\text { Refinement }\end{array}$ \\
\hline $\begin{array}{l}\text { T11 } \\
\text { T12 } \\
\text { T21 } \\
\text { T22 }\end{array}$ & $\begin{array}{l}\overrightarrow{0} \\
w \\
\vec{\omega} \\
\ddot{\omega}\end{array}$ & $\begin{array}{l}4.8 \\
4.8 \\
3.7 \\
3.7\end{array}$ & $\begin{array}{l}0.19 \\
0.19 \\
0.19 \\
0.19\end{array}$ & $\begin{array}{l}\text { No } \\
\text { Yes } \\
\text { No } \\
\text { Yes }\end{array}$ \\
\hline $\begin{array}{l}\text { M11 } \\
\text { M12 } \\
\text { M13 } \\
\text { M21 } \\
\text { M22 } \\
\text { M23 }\end{array}$ & 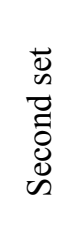 & $\begin{array}{l}4.8 \\
4.8 \\
4.8 \\
3.7 \\
3.7 \\
3.7\end{array}$ & $\begin{array}{l}0.04 \\
0.42 \\
1.08 \\
0.04 \\
0.42 \\
1.08\end{array}$ & $\begin{array}{l}\text { No } \\
\text { No } \\
\text { No } \\
\text { No } \\
\text { No } \\
\text { No }\end{array}$ \\
\hline
\end{tabular}




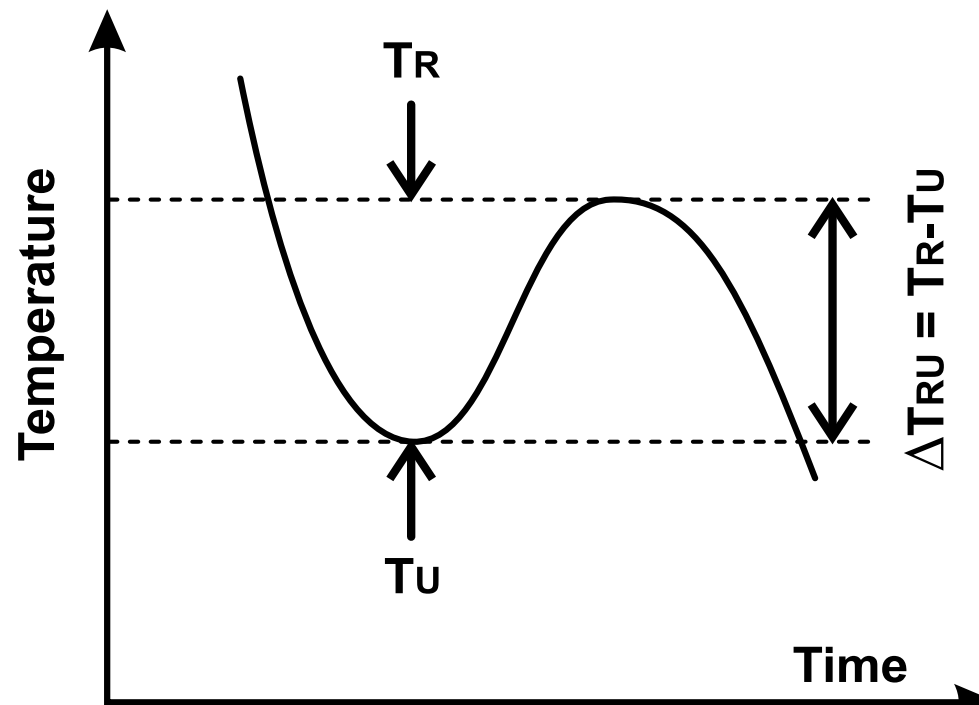

Figure 1. Undercooling criteria to examine the effectiveness of the grain refinement process

\section{Results and discussion \\ Microstructural Analysis}

Microstructure of the first set of experiments is presented in Fig. 2. For the case of no-refined samples the microstructure consists of very coarse grains and the dendritic morphology of $\alpha_{\mathrm{Al}}$. Refined samples, on the other hand, have a very fine equiaxed grain structure and no sign of dendrites can be seen in the structure. Generally at large grain sizes the grains are not equiaxed and tend to growth in certain crystallographic directions, but when growth is limited by either thermal or structural factors, equiaxed grain structure can be achieved (as can be seen in the structure of modified samples). The change in morphology may be interpreted in terms of the sever increase in active nucleation sites for $\alpha_{\mathrm{Al}}$ grains and/or the high $\mathrm{GRF}^{1}$ value $[9,14]$. Thus the number of $\alpha_{\mathrm{Al}}$ grains will increase and their impingement on the early stages of solidification reducing the chance of dendrite branching.

\footnotetext{
${ }^{1}$ Grain Restriction Factor
} 

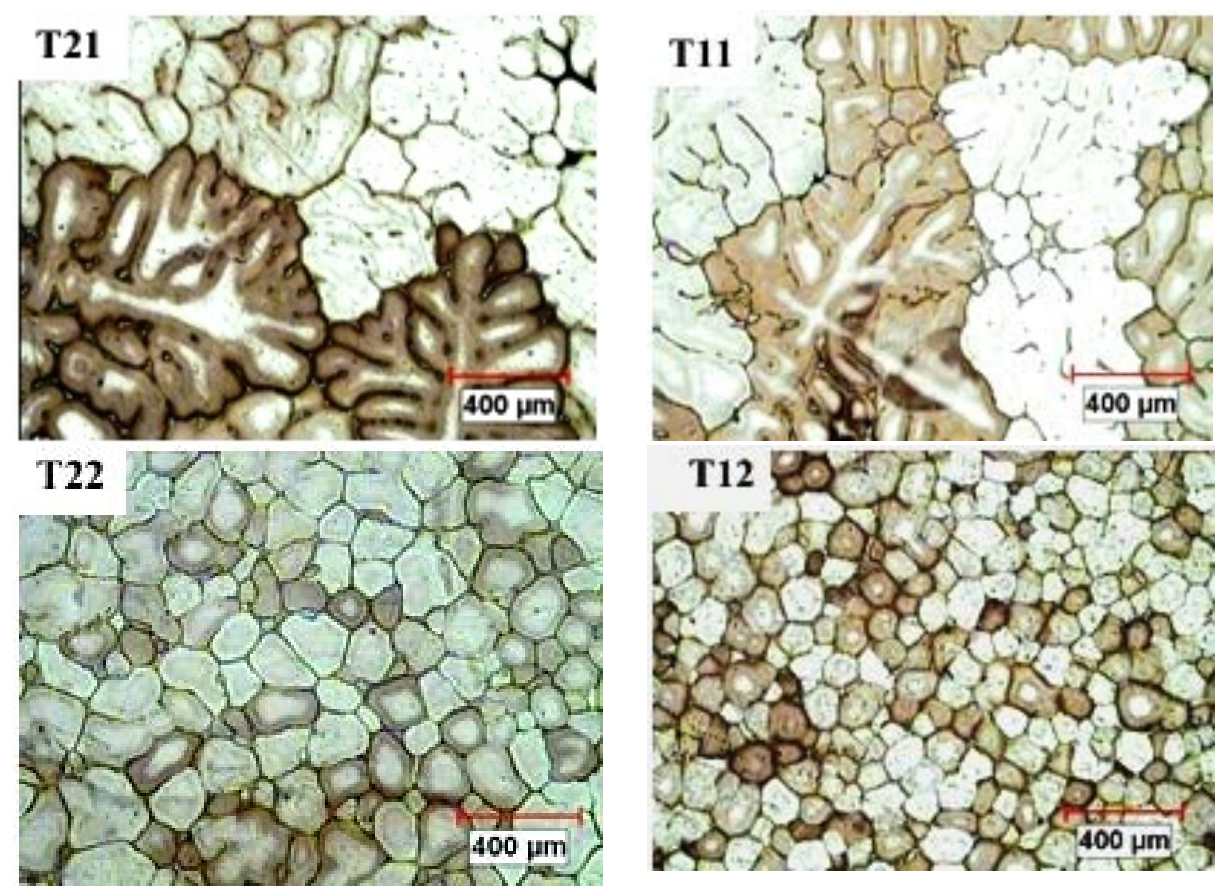

Figure 2. General microstructure of first set of samples.

Copper content for both alloys is less than the maximum solubility of copper at eutectic temperature $(5.65 \mathrm{wt} . \%)$ so it's expected that both alloys should be solidified with a single phase structure $\left(\alpha_{\mathrm{Al}}\right)$. The structure of this set of experiments with higher magnification is presented in Fig. 3. It's obvious that both alloys contain some eutectic phases which imply that the solidification process was non-equilibrium (NEq). The content of the NEq eutectic is usually considered as criteria for quantification of microsegregation level. As the NEq eutectic content increases the microsegregation level is also increases. It's clear that the NEq eutectic content for refined sample much less than the no-refined sample. So it can be concluded that the grain refinement reduces the microsegregation level. The main mechanisms which frequently discussed in the literature that affect the microsegregation are back diffusion and coarsening $[17,18]$. For both mechanisms the diffusion plays an important role in two ways: diffusion time and diffusion distance. Diffusion time is directly related to solidification time which was not affected by the grain refinement. So the only affective parameter is diffusion distance which is related to the scale of microstructure. By grain refinement the diffusion distance will reduce and hence the level of microsegregation reduces. Change the morphology of interface from the dendritic (for no-refined samples) to planar (for refined samples) will also affect the microsegregation. It's usually considered that the dendritic morphology shows the most microsegregation level [1]. 

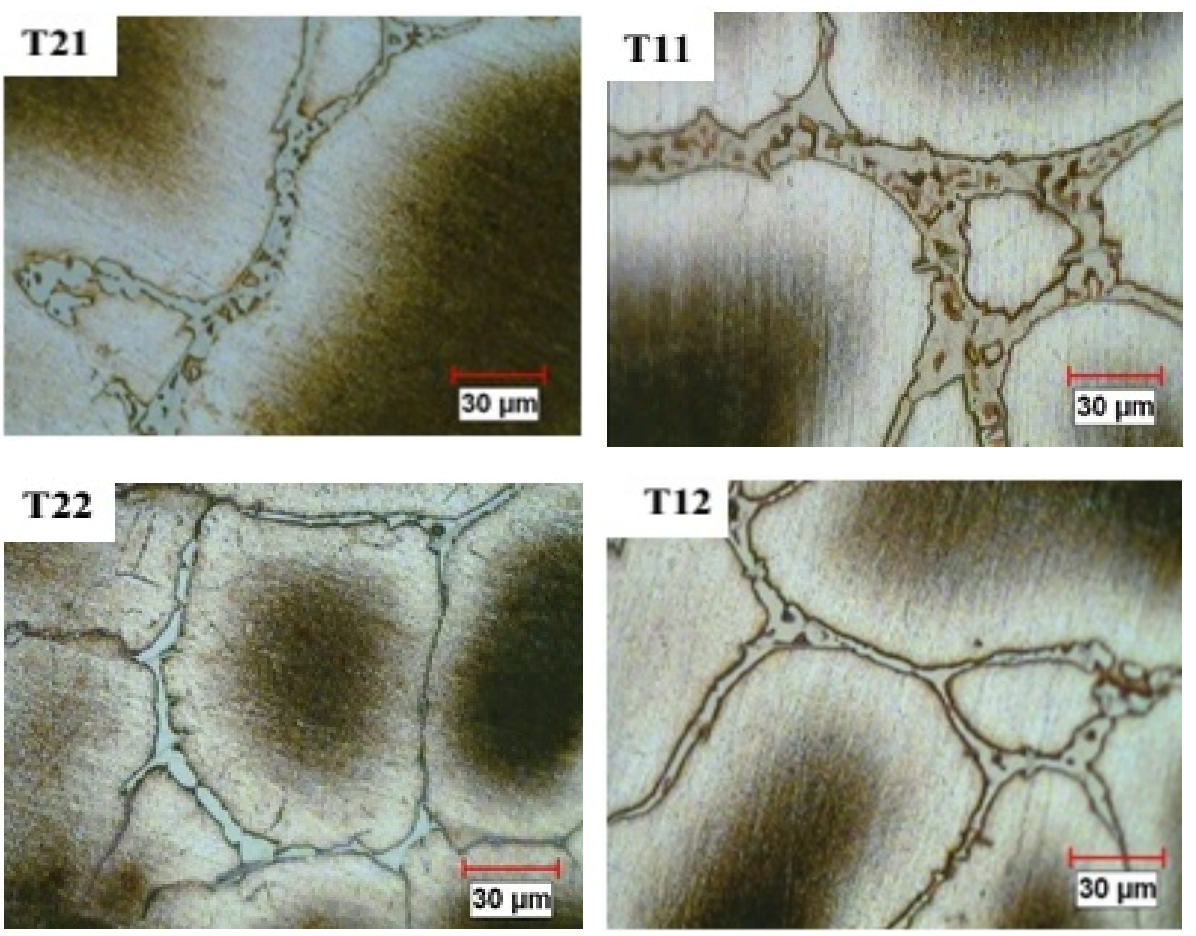

Figure 3. Eutectic structure and fraction of the first set of experiments (Weck's reagent).

Fig. 4, shows the SEM Backscattered Electron (BSE) images taken from the all samples. Two types of phases are shown in this region, since the contrast of BSE image is mainly from the mean atomic number, the phase with a higher mean atomic number shows up brighter in the BSE image. The dark phase is primary $\alpha_{\mathrm{Al}}$ and $\mathrm{Al}_{2} \mathrm{Cu}$ phase (white phase, due to its relatively high $\mathrm{Cu}$ concentration) precipitated from the $\alpha$-phase and normal eutectic microstructure. The $\alpha-\mathrm{Al}_{2} \mathrm{Cu}$ phase distributed uniformly but discontinuously in the interdendritic regions. It is clear that with grain refinement alloy the content of NEq eutectic (bright phase) decreases.
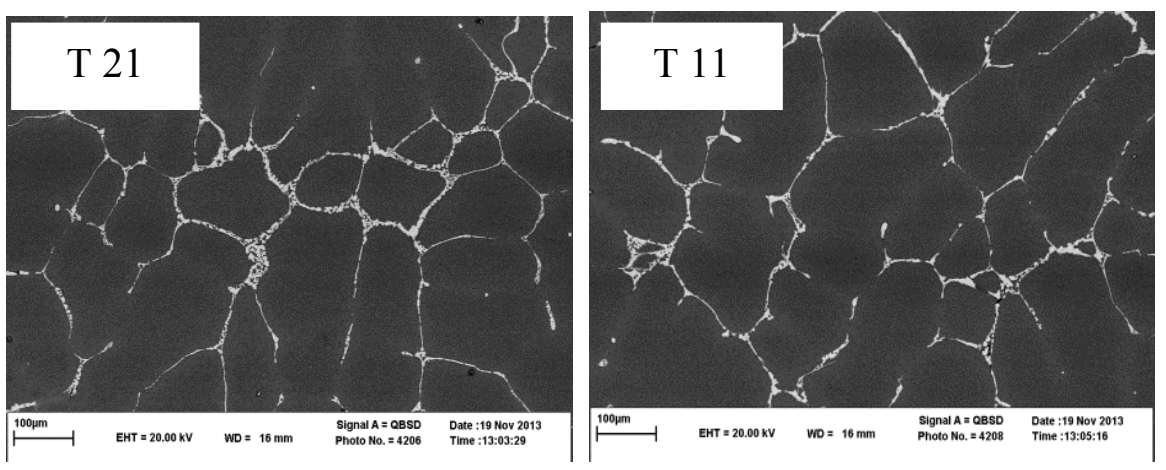

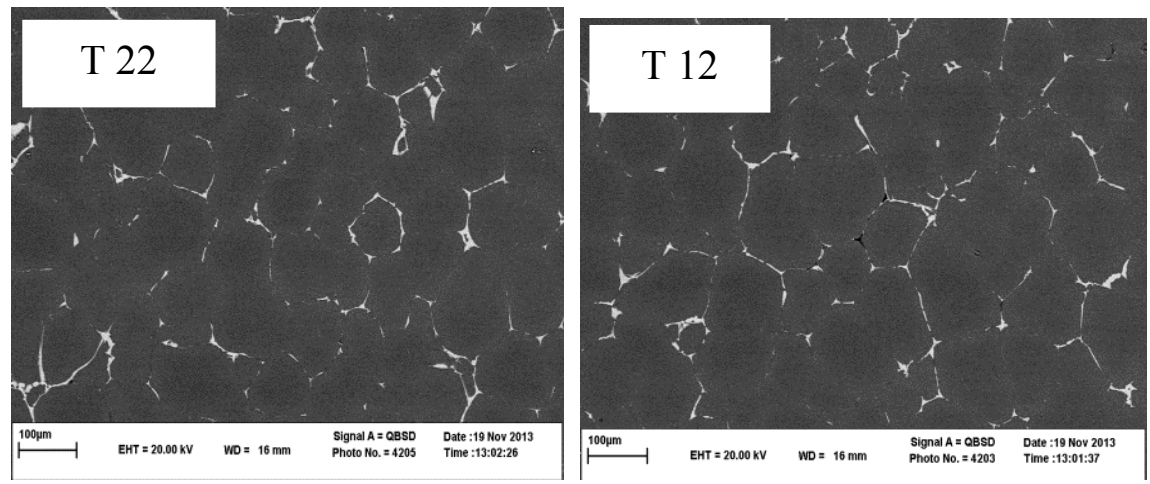

Figure 4 . BSE migrographs of the samples.

\section{Thermal Analysis}

The cooling curve and its first derivate curve for the T11 and T12 samples are presented in Fig. 5. According to cooling an derivative curves it can be seen that the solidification are consisted of two steps: at first step the solidification starts by development of a dendritic network. First inflection point on the curves results from the evolution of the heat of solidification of the $\alpha_{\mathrm{Al}}$. In the second step at low temperatures, however, solidification followed by a eutectic reaction. During solidification two phenomena occur simultaneously, i.e. nucleation and growth. As the temperature decreases (increasing the level of undercooling) the nucleation rate rises and at the same time, growth of the formed nucleus will release the latent heat of solidification which reduces the cooling rate until it reaches to a minimum temperature; Recalescence temperature. Thereafter the temperature rises again to its normal growth temperature due to release of more latent heat. After the end of recalescence, the nucleation process is completed and no more new $\alpha_{\mathrm{Al}}$ particles will form [19].

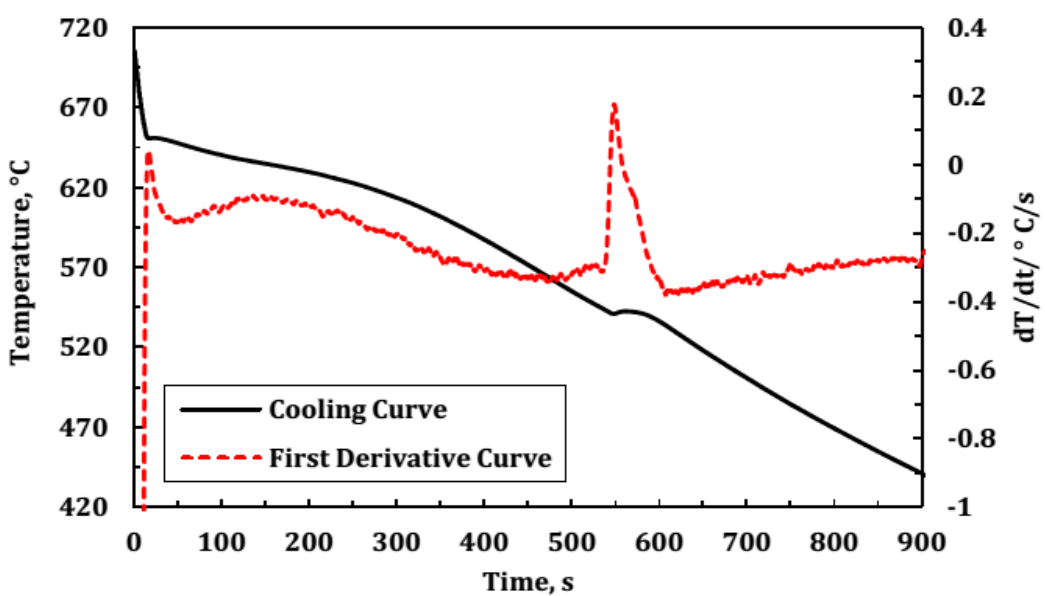




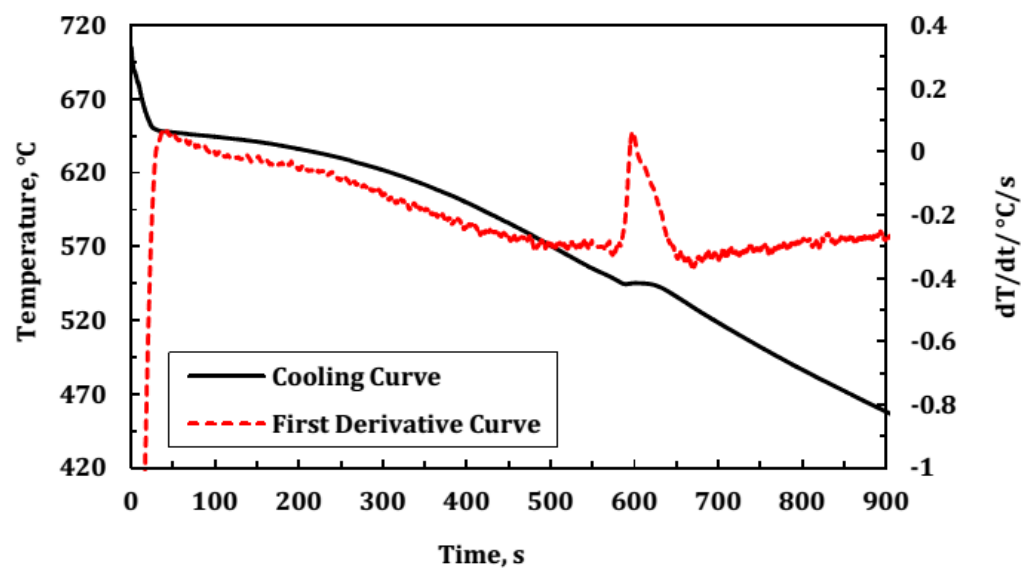

Figure 5. Cooling curves and first derivative for samples T11 and T12

The occurrence of the recalescence depends on the density of the nucleation sites. If the melt is no grain refined, a greater driving force in the form of undercooling will be necessary to start solidification of the primary grains. Once nucleation is completed, evolution of latent heat tends to decrease the undercooling and recalescence occurs. The apparent undercooling then is defined as the difference between the minimum temperature relating to the beginning of solidification and the maximum temperature reached by the alloy during solidification. This apparent undercooling, $\Delta \mathrm{T}_{\mathrm{RU}}$, is shown in Fig. 1. The beginning of cooling curve for T11 and T12 is shown in Fig. 6. It can be seen that the grain refinement affects the shape of the cooling curve at the beginning of solidification. In T12 sample, for example, with a sufficient number of effective nuclei, nucleation will occur in a shorter time. This indicates that there is almost no energy barrier for nucleation and that the grain size of the casting will be fine [13]. The shape of the cooling curve at the beginning of the solidification process gives a good indication of the number of nuclei present in the melt. When there are a great number of nuclei, the curve exhibits low undercooling, as illustrated in the figure. When there are few nuclei, a higher extent of undercooling may be expected as can be seen for T11 sample. This figure clearly shows that the melt needs to be undercooled before nucleation of new $\alpha_{\mathrm{Al}}$ particles occurs. It should be noted that $\Delta \mathrm{T}_{\mathrm{RU}}$ does not represent the undercooling $\Delta \mathrm{T}$, required for the nucleation of solid. $\Delta \mathrm{T}_{\mathrm{RU}}$ is only the point at which heat loss from the sample becomes less than the rate of heat generation from latent heat evolution [20]. 


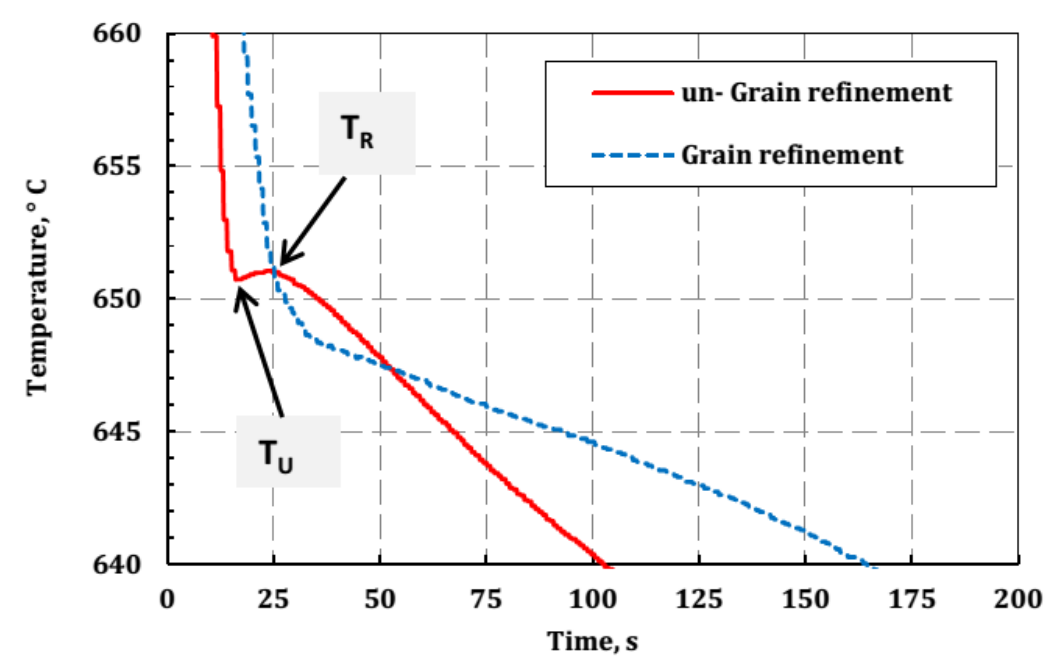

Figure 6. Comparison between the cooling curves of T11 and T12 at the beginning of the solidification

\section{Effect of Inoculation on Eutectic Undercooling}

As shown in the metallographic investigation, grain refinement also affects the volume fraction of $\mathrm{NEq}$ eutectic. This effect maybe better understood using undercooling concept. Although the liquidus temperature of the alloys have changed by inoculation, the results also showed that it has more pronounced effect on eutectic undercooling and volume fraction of non-equilibrium eutectic. Eutectic range is defined as temperature and time difference between start of eutectic transformation and solidification completion $\left(\Delta T_{E}=T_{E}-T_{S}, \Delta t_{E}=t_{E}-t_{S}\right)$. Fig. 7. shows the eutectic range for samples T21 and T22. It can be seen that for the sample T21 eutectic undercooling decreases from $\Delta T_{E}=7.2$ to $3.3^{\circ} \mathrm{C}$ for $\mathrm{T} 22$. The eutectic time range $\left(\Delta t_{E}\right)$ decreases from 43 to $25 \mathrm{~s}$. Table 3 . shows the results of thermal analysis of the first set of experiment. 


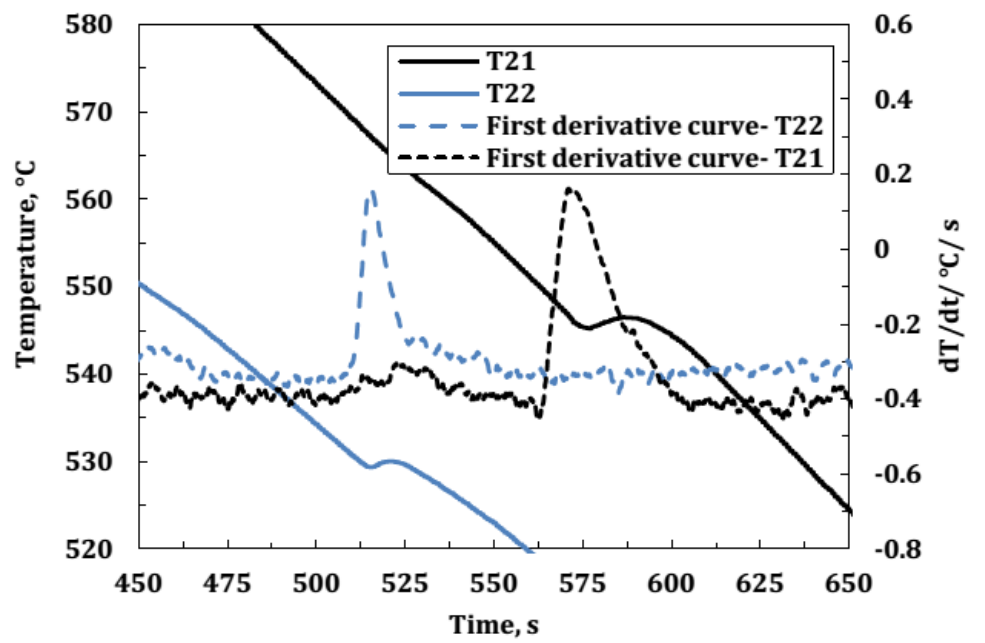

Figure 7. End of the solidification portion of cooling curve for the samples T21 and T22.

Table 3. Results of thermal analysis for first set of experiments

\begin{tabular}{cccccc}
\hline Sample Code & $T_{L}$ & $T_{E}$ & $T_{S}$ & $\Delta T_{E}$ & $\Delta t_{E(S)}$ \\
\hline$T 11$ & 651.3 & 543 & 533 & 10 & 65 \\
$T 12$ & 649.6 & 535.4 & 531 & 4.4 & 53 \\
$T 21$ & 653.1 & 546 & 538.8 & 7.2 & 43 \\
$T 22$ & 651.8 & 533.3 & 530 & 3.3 & 25 \\
\hline
\end{tabular}

\section{Solid Fraction vs. Temperature}

Solid fraction vs. temperature for the first set of experiments is calculated based on the Scheil equation (Eq. (6)) and plotted in Fig. 8. As can be seen, the solidification rate $(d f / d T)$ is very high at the beginning of solidification and it will decrease at the end of solidification. So a large fraction of melt solidifies at the early stage of solidification and the solidification rate slows down by decreasing the temperature so that the solid fraction reaches to $90 \%$ at eutectic temperature. The curves are very consistent at the early stages of solidification (up to $80 \%$ solid) but at the end of solidification the deviation is increased especially because the microsegregation is more effective at the end of solidification. 

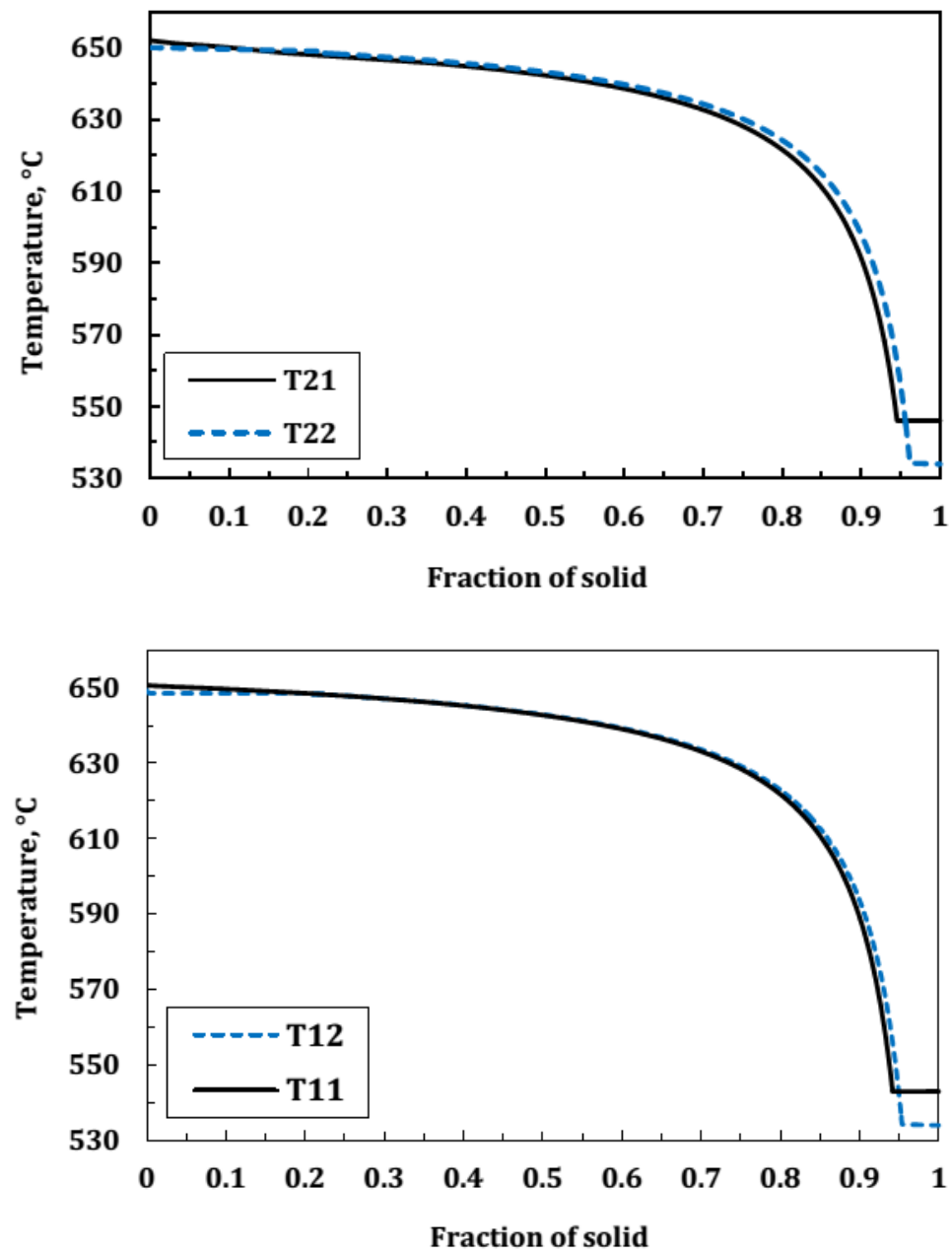

Figure 8. Solid fraction vs. temperature for first set of experiments

\section{Calculation of Latent Heat of Solidification}

These calculations were just performed on the second set of the experiments which were examined in DTA furnace. As is implied by the name zero curve, i.e., a curve without phase transformation, only the portion of data that is not affected by the latent heat of solidification should be used in the calculation. There are variety of methods to calculate the zero curve but the best way is to accurately determine the beginning and the end points (temperatures and times) of solidification and draw a line between these two points [7]. The first derivative curve has been used to identify the exact points for the beginning and the end of solidification. Fig. 9. shows the cooling curve, its first derivative, and calculated Newtonian zero curves for sample M12. Here only 2 data points (beginning and the end of solidification), which were precisely 
determined with the aid of first derivative cure, were used. The same way was used to calculate the zero curve for other samples. Table 4. shows the used solidification characteristics of this set of the experiments.

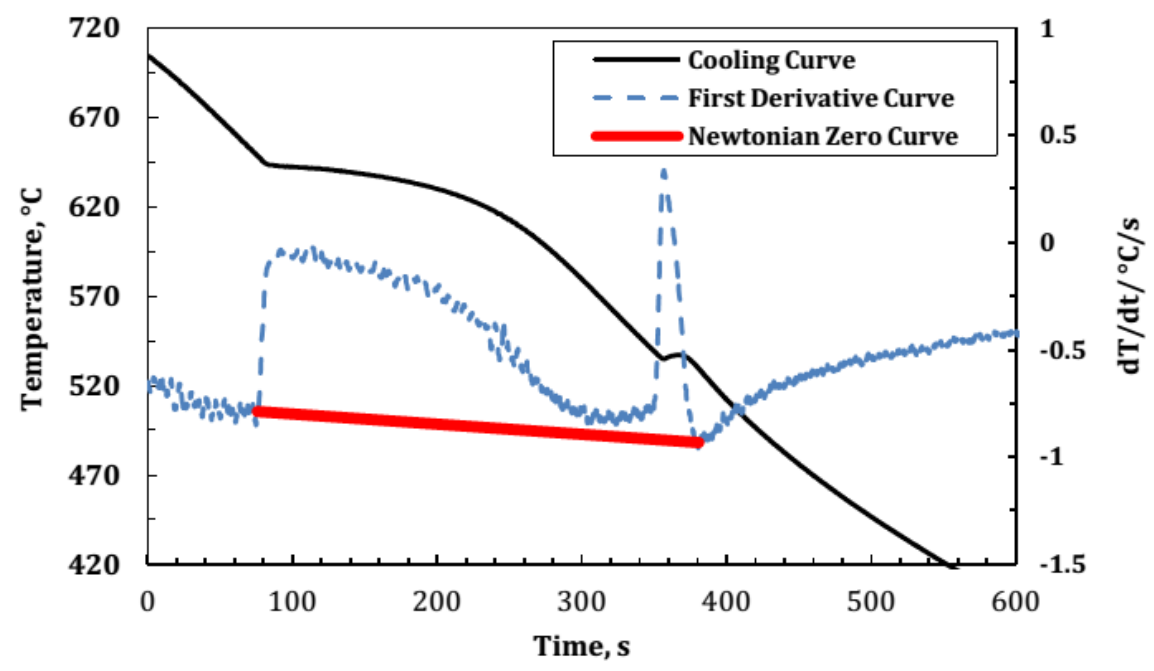

Figure 9. Zero curve calculation by linear fitting from liquidus to solidification end point for $M_{12}$ sample.

Table 4. Thermal characteristics of the second set of experiments

\begin{tabular}{ccccc}
\hline Sample Code & $T_{L}{ }^{\circ} \mathrm{C}$ & $T_{S}{ }^{\circ} \mathrm{C}$ & $t_{L(s)}$ & $t_{S(s)}$ \\
\hline M11 & 650 & 542 & 1128 & 4021 \\
M12 & 648 & 531.5 & 78 & 380 \\
M13 & 646 & 529 & 28 & 134 \\
$M 21$ & 652 & 544 & 1420 & 4125 \\
$M 22$ & 650 & 536 & 57 & 348 \\
$M 23$ & 648 & 531 & 34 & 146
\end{tabular}

In order to calculate the latent heat of solidification the specific heat as a function of temperature for both liquid and solid is required in Newtonian method. Backerud claimed that the difference in specific heat of the solid and liquid is so small that a same value can be used for both solid and liquid [21]. Accurate calculations of latent heat largely depend on the accurate calculation of the zero curve and a proper choice of specific heat. In determining the specific heat value, since we are interested in the region from liquidus to the solidification end point, a better option is to use a value of specific heat between liquid and solid for a mushy state. An average value of $1.078 \mathrm{~J} / \mathrm{g} . \mathrm{K}$ was selected from the literature and used in these calculations. To calculate latent heat, first the area between the first derivative and the zero curve were calculated 
by numerical integration and then latent heat was found by multiplying this value by specific heat. The results are presented in Table 5. Based on the results, it can be seen that there is clear difference between the values calculated at high cooling rates compared to those calculated at low cooling rates. Some part of the difference can come from this fact that Newton's method was assumed that the thermal gradients across the sample can be ignored which this assumption may not be satisfied at high cooling rates. It can also because the fact that the latent heat of solidification is depend on the cooling rate $[22,23]$.

\section{Conclusions}

In this study, the effect of cooling rate and grain refinement on microstructure, thermal and thermo-dynamic properties of binary Al-Cu alloys by Newtonian thermal analysis was studied. The following results were obtained:

1- With Considering the changes in the primary undercooling $\left(\Delta \mathrm{T}_{\mathrm{RU}}\right)$ as the main factor to determine the effectiveness of grain refinement process, it was found that by grain refinement, the value of undercooling decrease approximately to zero.

2- The results of microstructure and thermal analysis showed that the main effect of grain refiner is on eutectic solidification range. It will reduce the eutectic solidification range and volume fraction of $\mathrm{NEq}$ eutectic in the final structure.

3- Grain refinement reduces the degree of microsegregation by reducing the NEq eutectic content.

4- The calculated values of the latent heat was in good agreement with the results of previous works especially for low cooling rates. But the uncertainity will increase as the cooling rate increases.

The sample cooled in the slow cooling range (around $0.1 \mathrm{~K} / \mathrm{s}$ ) is suitable for calculation value of latent heat by Newtonian's and by incresase cooling rate the results of this method is not very accurate.

\section{References}

[1] B. S. Murty, S. A. Kori, And M. Chakraborty, International Materials Reviews, 47 (2002) 4-29.

[2] C. Limmaneevichitr, W. Eidhed, Mater. Sci. Eng. A,349 (2003) 197-206.

[3] M. Malekan, S. G. Shabestari, J Therm Anal Calorim, 103 (2011) 453-458.

[4] S. L. Backerud, G.K. Sigworth, Afs Trans, 97 (1989) 459-464.

[5] D. Emadi, J Therm Anal Calorim,81 (2005) 235-242.

[6] O. Fornaro, H.A. Palacio, J. Mater. Sci,44 (2009) $4342-4347$.

[7] Ihsan. Ul. Haq, J. S. Shin, Z. H. Lee, Met. Mater. Int,10 (2004) 89-96.

[8] J.O. Barlow, D.M. Stefanescu, Afs Trans, 105 (1997) 349-354.

[9] J. W. Gibbs, P. F. Mendez, Scripta Materialia, 58 (2008) 699-702.

[10] W.T. Kierkus, J.H. Sokolowski, Afs Trans, 66 (1999) 161-167.

[11] D. Emadi, L. Whiting, M. Djurdjevic, W.T. Kierkus, J.H. Sokolowski, Metalurgija,( 2004) 91-106.

[12] D.M. Stefanescu, G. Upadhya, D. Bandyopadhyay, Metall. Trans A, 21(1990), 997-1005.

[13] H. Li, T.Sritharan, Y.M. Lam, N.Y. Leng, J. Mater. Proces. Tech, 66 (1997) 253257. 
[14] Y.Birol, Int. J. Cast Met. Res, 25 (2012) 117-120.

[15] M. Krupinski, K. Labisz, L.A. Dobrzanski, Z.M. Rdzawski, Journal of Achievements In Materials And Manufacturing Engineering, 38 (2010) 115-121.

[16] S.T. Yeh, Proceedings of The Twenty-Seventh Annual SAS User Group International (SUGI) Conference, 27 (2002) 229-27.

[17] D. Eskin, Q. Du, D. Ruvalcaba, L. Katgerman, Mater. Sci. Eng. A, 405 (2005) 110.

[18] Q. Du, D.G. Eskin, A. Jacot, L. Katgerman, Acta Mater, 55 (2007) 1523-1532.

[19] M. Dehnavi, M. Haddad Sabzevar, Metall. Mater. Eng. 20 (2014) 183-190.

[20] M.M. Isfahani, P.H.D Thesis Mc Gill University, (1995) 89-91.

[21] L. Backuerud, G. Chai, J. Tamminen, Foundry Alloys, Afs/Skanaluminium, Stockholm, Sweden, (1990) 255.

[22] J. Mahmoudi, H. Fredriksson, Mater. Sci. Eng. A, 226-228 (1997) 22-27.

[23] J. Mahmoudi, H. Fredriksson, J. Mater. Sci,35 (2000) 4977-4987.

[24] A.E. Ares, S.F. Gueijman, C.E. Schvezov, Metals And Materials Society, (2000) 615-621.

[25] C.Y. Wang, C. Beckermann, Metals And Materials Society, (1994) 135-141

[26] S. Ruben, Handbook of The Elements, La Salle, Illinois: Court Publishing Company, (1984) 59-102. 